\title{
Electrical properties of MIS structures with silicon nanoclusters
}

\author{
S.V. Bunak ${ }^{1}$, V.V. Ilchenko ${ }^{1}$, V.P. Melnik ${ }^{2}$, I.M. Hatsevych ${ }^{2}$, B.N. Romanyuk ${ }^{1}$, \\ A.G. Shkavro' ${ }^{1}$, O.V. Tretyak ${ }^{1}$ \\ ${ }^{1}$ Taras Shevchenko Kyiv National University, Institute of High Technologies \\ 64, Volodymyrska str., 01601 Kyiv, Ukraine \\ ${ }^{2}$ V. Lashkaryov Institute of Semiconductor Physics, NAS of Ukraine \\ 41, prospect Nauky, 03028 Kyiv, Ukraine \\ E-mail: ilch@univ.kiev.ua
}

\begin{abstract}
The theoretical and experimental investigations of electrical properties of the $\mathrm{Al}-\mathrm{SiO}_{2}-(\mathrm{Si}-\mathrm{ncs})-\mathrm{SiO}_{2}-\mathrm{Si}$ structures grown using high temperature annealing $\mathrm{SiO}_{x}$, $x<2$, have been carried out. It has been experimentally found that the $\mathrm{Al}-\mathrm{SiO}_{2}$ $(\mathrm{Si}-\mathrm{ncs})-\mathrm{SiO}_{2}-\mathrm{Si}$ structures with the tunnel dielectric layer revealed the effect of dynamic memory. Electric properties and parameters of the interface states located between $\mathrm{Si}-$ ncs and $\mathrm{SiO}_{2}$ were studied in detail by measuring of current-voltage, capacitance-voltage, and thermally stimulated current characteristics.
\end{abstract}

Keywords: nanocluster, static conductance, dynamic conductance, hopping transport, thermally stimulated current, memory effect..

Manuscript received 11.01.10; accepted for publication 16.03.11; published online 30.06.11.

\section{Introduction}

Intensive investigations of metal-insulator-silicon (MIS) structures with various nanoobjects, such as quantum dots, quantum wires, quantum rings, and quantum holes have been recently performed. One of the most promising MIS structures with silicon nanoclusters in insulator attracted particularly great attention as concerning the development of nanomemory devices. This kind of structures reveals new specific features in their differential capacitance, which opens qualitatively new opportunities to develop dynamic memory devices on their base $[1,2]$. Besides, $\mathrm{Al}_{-}-\mathrm{SiO}_{2}-(\mathrm{Si}-\mathrm{ncs})-\mathrm{SiO}_{2}-\mathrm{Si}$ structure, owing to its atypical differential capacitancevoltage characteristics $(C-V)$ can work as a separate functional device, which can replace the whole circuits and units by itself.

In most works where the structures $\mathrm{Al}-\mathrm{SiO}_{2}$ $(\mathrm{Si}-\mathrm{ncs})-\mathrm{SiO}_{2}-\mathrm{Si}$ were studied, main attention was paid to quantum-sized effects in nanoclusters. At the same time, only a few attention was paid to researching the influence of interface states on electrical properties of these structures.
In this work, we studied the influence of interface states related with nanoclusters on electrical properties in the structures $\mathrm{Al}-\mathrm{SiO}_{2}-(\mathrm{Si}-\mathrm{ncs})-\mathrm{SiO}_{2}-\mathrm{Si}$.

\section{Preparation of samples}

The experimental samples were prepared as a layered MOS structure containing $\mathrm{Si}$ nanoclusters in $\mathrm{SiO}_{2}$ on the previously oxidized $p-\mathrm{Si}$ in dry $\mathrm{O}_{2}$ atmosphere at the temperature $700{ }^{\circ} \mathrm{C}$ for $10 \mathrm{~min}$ on $p-\mathrm{Si}$ substrate (doped with B, $N_{A}=4 \times 10^{14} \mathrm{~cm}^{-3}$ ). The detailed scheme of the investigated samples is shown in Fig. 1. Then the $\mathrm{SiO}_{x}$ layer with $x<2$ (thickness $40 \mathrm{~nm}$ ) was grown. After that, another thermal processing was done at the temperature $1150{ }^{\circ} \mathrm{C}$ in nitrogen atmosphere for $10 \mathrm{~min}$. After phase reconstruction, $\mathrm{Si}$ nanoclusters with sizes close to $4 \mathrm{~nm}$ were created in $\mathrm{SiO}_{2}$ layer.

\section{Experimental results and discussion}

Behaviour of structures with nanoclusters in insulator obtained using high-temperature annealing of $\mathrm{SiO}_{x}$ with 
$x<2$ technology [3-5] (Fig. 1) was studied in this work.

Electrophysical parameters of these structures in high-frequency equilibrium and non-equilibrium $C-V$ characteristics and dependences of differential conductivity on the applied voltage in a wide range of temperatures were investigated in detail.

Temperature dependences of $I-V$ curves were analyzed to determine the mechanisms of charge carrier transport. Then the method of thermally stimulated current (TSC) was used for characterization of trap energy levels at the boundary of nanoclusters.

\section{1. $C-V$ characteristics}

The experimental investigation of capacitance-voltage characteristics was carried out using the automated installation [6]. The block-scheme of the latter is shown in Fig. 2.

The investigated samples were placed into the thermostat which could provide temperature variation from $-77 \mathrm{~K}$ up to $450 \mathrm{~K}$ with a good accuracy. To measure admittance of the structure, it has been connected by four electrodes that were also placed into the thermostat. The minimal voltage step applied to the sample was $0.005 \mathrm{~V}$. The applied testing signal was modulated by the frequency $10 \mathrm{kHz}$ with the amplitude $0.04 \mathrm{~V}$.

The thermostabilization system consisting of the block for controlling the thermostat was operated by a power unit, ADC/DAC converters and software. The temperature was measured by the thermode supplied by a source of current being in the thermostat controlling block. The data voltages from the thermode were treated by ADC. All these data were read out by computer program and transformed into the value of temperature using the formulae of recalculation delivered by the manufacturer of this thermode. Using the program with specially designed algorithm of thermostabilization, the corresponding signal generated by a separate power unit was applied to the heater in the thermostat. Depending on the value of applied voltage, the range of temperature stabilization could be provided as $0.01{ }^{\circ} \mathrm{C}$.

All measurements of the sample parameters were carried out using RLC measuring instrument E7-14, Electrometer Keithley 6485. The bias voltages were taken from the specially designed DAC sockets to adjust their output parameters with requirements of the input interface of the RLC measuring instrument E7-14. Used for the current measurements was B7-46 device switched in the corresponding mode.

Since all the measuring instruments were supplied with the IEEE488 interfaces, a specially designed computer program containing IEEE488 interface module controller was used for automatic controlling the measurements. The designed computer program contained necessary number of additional functions for the previous mathematic and statistic treatment of the experimental results.

\begin{tabular}{|c|}
\hline Metal contact (Al) \\
\hline $\mathrm{SiO}_{2}$ containing Si nanoclusters $(40 \mathrm{~nm})$ \\
\hline $\mathrm{SiO}_{2}(4.7 \mathrm{~nm})$ \\
\hline -Si $\quad p=4 \times 10^{14} \mathrm{~cm}^{-3}$ \\
\hline InGa Ohmic contact
\end{tabular}

Fig. 1. Scheme of the investigated samples.

In the course of these measurements, it was clearly ascertained that the structure with $\mathrm{Si}$ nanoclusters demonstrated properties atypical for ordinary MIS structures as to partial accumulation of the charge in the electron states located in close proximity to $\mathrm{Si}$ nanoclusters. It has been noted that the investigated structure shows the relaxation time that could achieve several hours. That is why all the $C-V$ (capacitancevoltage) and $G-V$ (conductance-voltage) measurements presented in this paper could be performed in stationary and dynamic modes with rather slow RLC measuring instruments such as E7 - 14 .

Stationary $C-V$ and $G-V$ measurements were carried out with the step $0.1 \mathrm{~V}$ and the time interval between measurements $100 \mathrm{~s}$. Before measurements, the structures were kept under the initial reverse bias voltage $(-10 \mathrm{~V})$ for several hours to provide conditions when all electron states near the nanoclusters could come to their definite stationary state.

Dynamical $C-V$ and $G-V$ characteristics were obtained in the following way: the regime voltage applied to the structure for a relatively short time until the relaxation processes in the investigated structure would not be sufficient. Thus, the measurements of capacitance and conductivity were carried out in the following sequence: set of the required bias voltage was done first, then after a short idle time (about $1 \mathrm{~s}$ ) the measurements of characteristics were performed. The delay between setting the voltage and measurements could be changed and was made long enough in order to all quick radiotechnical transient processes could have come to the end.

After measurements, the regime voltage was applied to the structure for its relaxation to the initial state. The next measurement was performed after the time period (about $5 \mathrm{~s}$ ), which would be necessary kept for the relaxation to the some regime state. An exact value of relaxation time could be experimentally obtained on the grounds of the following reasoning: the form of the measured dependence should not change because of insufficient times of relaxations and also time necessary for carrying out the measurements. 


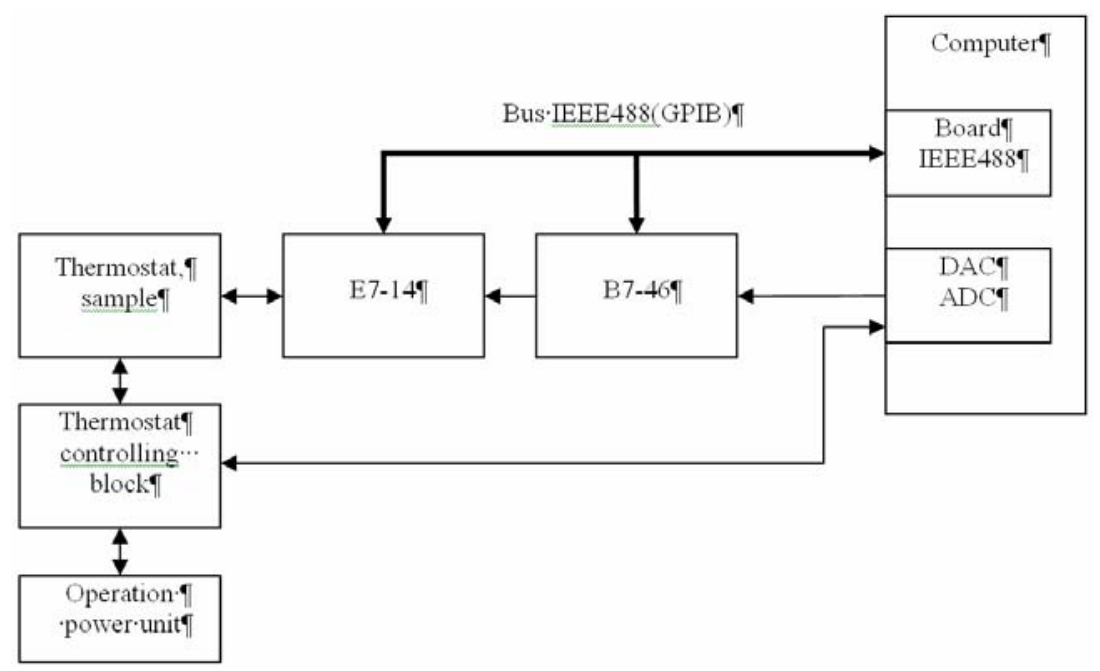

Fig. 2. Block diagram of the installation.
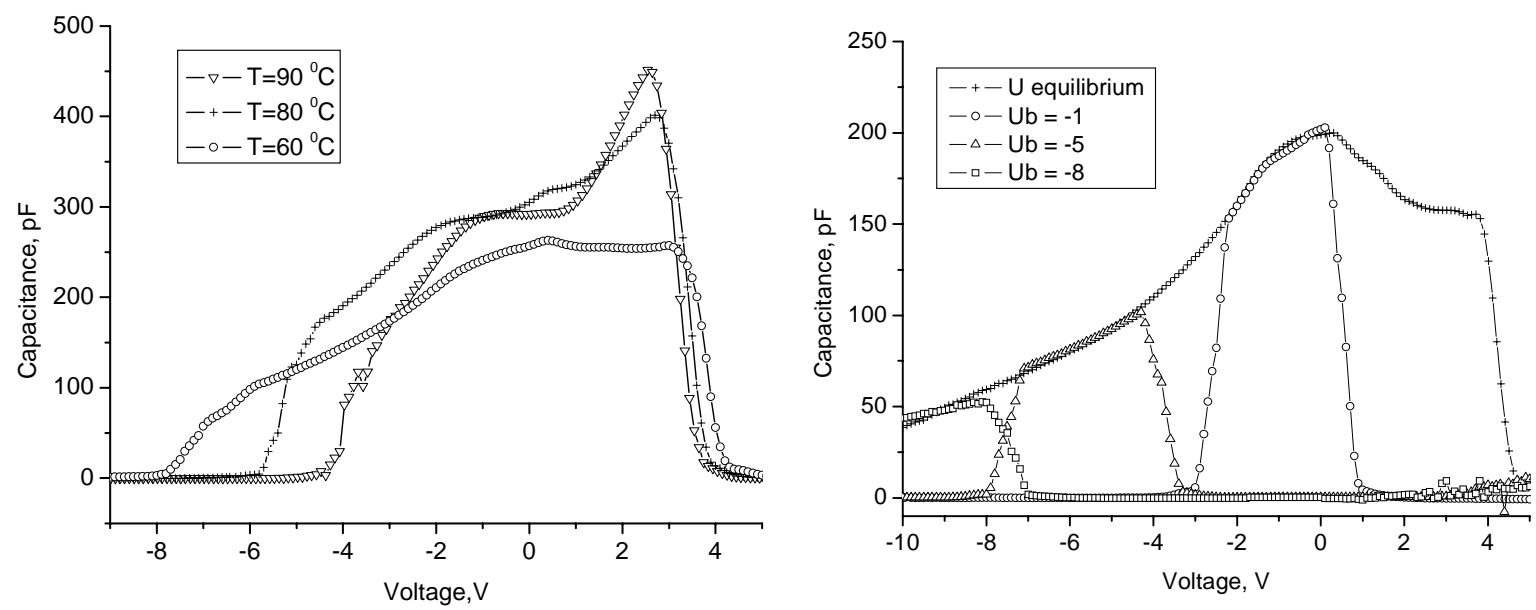

Fig. 3. a) Stationary $C-V$ curves taken for different temperatures and b) stationary (equilibrium) and dynamic $C-V$ curves taken at $293 \mathrm{~K}$.

The results of measurements performed in the dynamic mode are shown in Fig. 3. Adduced there are features of stationary $C-V$ characteristics with their atypical behaviour in comparison with standard characteristics of MIS structures presented.

The growth of stationary high-frequency capacitance begins with some voltage, which essentially depends on the temperature of samples. At high temperatures, the capacitance grows relatively fast. In the meantime for low temperatures, the capacitance begins to grow considerably earlier even at reverse bias (see Fig. 3a). Falling down inherent to $C-V$ curves occurs at positive bias voltages, which is quite identical for all the dependences, and it did not practically depend on temperature. With the growth of temperature, the maximum of dependences for $C-V$ characteristics becomes more clearly pronounced.

In the case of dynamic $C-V$ characteristics, the value of capacitance essentially differs only in very definite range of the regime voltage component, whereas for other voltages the capacitance practically equals to zero. It is a typical feature of dynamic $C-V$ characteristics measured at a constant temperature with different regime voltages. It should be noted that outlined curves obtained for a series of dynamic characteristics repeated very precisely the stationary $C-V$ characteristics in Fig. 3b. This kind of $C-V$ behaviour can be well explained as caused by charging accumulation in the interface states at the boundary of nanoclusters.

Band-energy diagram of the MIS structure with $\mathrm{Si}$ nanoclusters is presented in Fig. 4. As it was described in [7], the superfluous atom of silicon could serve as the main reason for formation of energy traps in the insulator bulk. Besides, the surface of nanoclusters should be surrounded by the interface states. Here we suppose that energy levels of the interface states in nanoclusters are placed inside the silicon band-gap. 


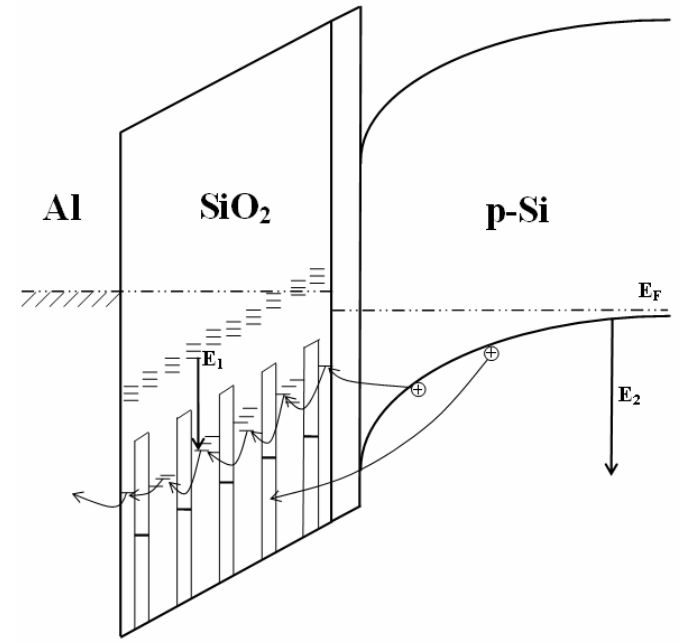

Fig. 4. Band-energy diagram of the MIS structure with Si nanoclusters.

During the measurements of $C-V$ curves, the scanning voltage fills the energy levels of the interface states with carriers (Fig. 4). For the high enough negative regime voltage, the Fermi level is moved outside the levels formed by the interface states, thus the high-frequency capacitance of the structure is equal to zero. With reduction of the reverse bias voltage, the Fermi level moves to the area of levels belonging to the interface states again. At slow changing the scanning voltage, the interface states relax in time.

The charge captured to the interface states was successfully investigated measuring the high-frequency capacitance, when sufficiently deep levels cannot follow the testing signal. With changing the bias voltage, the Fermi level position moves relatively to the energy position of interface states, and filling them with carriers is also changed, as a consequence. In this way, it is possible to explain that the investigated capacitance is falling off to zero gradually, when the scanning voltage reaches the certain value (for the given structure, it is close to $4 \mathrm{~V}$ (see Fig. 3a)) because the Fermi level leaves the belt of energy levels uniformly distributed in $\mathrm{SiO}_{2}$ and belonging to interface states.

When measuring the dynamic $C-V$ characteristics, the structure comes up to the dynamic equilibrium condition corresponding to very definite regime voltage value. As the processes of charge capture to the energy levels of interface states go slowly, filling these levels is mainly dependent on the regime voltage changing rate. If the scanning voltage leaves the certain range of values faster than the charge in interface states can be changed, the differential capacitance measured at $10 \mathrm{kHz}$ testing signal becomes equal to zero. Otherwise $C-V$ dependences repeat the curve obtained in the stationary mode measurements. Thus, the scanning voltage may be set in two different regimes when the interface states are empty or filled. In those cases, the dynamic $C-V$ characteristics measured with a sufficiently small step of direct voltage value can form the behaviour identical to the stationary $C-V$ characteristics. Gathering together $C-V$ characteristics obtained for the series of dynamic measurements, the stationary $C-V$ dependence can be rebuilt as their enveloping curve (Fig. 3b).

As it is clearly follows from the experimental results, the differential capacitance of the structure containing silicon nanoclusters in insulator essentially depends on a charge accumulated in the interface states that are formed at the boundaries between $\mathrm{Si}$ nanoclusters and $\mathrm{SiO}_{2}$. The dynamic researches of the capacitance show that the charge on the interface states can be kept for a sufficiently long time (in investigated samples influence was observed within several hours). Being based on the experimental results, one can make a conclusion that the investigated structures clearly and reproducibly show the specific feature of dynamic memory effect.

\subsection{Current investigation}

\subsubsection{Investigation of $I-V$ characteristics}

The temperature dependences of $I-V$ curves were taken within a rather wide temperature range ($60{ }^{\circ} \mathrm{C} \ldots+20^{\circ} \mathrm{C}$ ) for the forward and reverse biases. The results of these measurements have been presented in Figs $5 \mathrm{a}$ and $5 \mathrm{~b}$.

With decreasing the temperature, $I-V$ curves correspond to the structure with properties of slightly rectifying contacts. There are two different parts of the characteristics with two different activation energies: $E_{1}=0.3 \mathrm{eV}$ for the reverse and small forward biases; $E_{2}=0.6 \mathrm{eV}$ for the large enough forward bias. The plots providing calculation of the activation energy values are presented in the inserts to Figs $5 \mathrm{a}$ and $5 \mathrm{~b}$.

As it was described for the similar case [7], the main transport mechanisms in the structures with $\mathrm{Si}$ nanoclusters in $\mathrm{SiO}_{2}$ are the hopping ones through the chains of superfluous $\mathrm{Si}$ atoms inside $\mathrm{SiO}_{2}$. At the same time, the boundary of $\mathrm{Si}$ nanoclusters contains a considerable quantity of interface states, where a very noticeable electric charge can be accumulated. To achieve the dynamic equilibrium, carriers should refill all these states when the quasi-Fermi level is shifted by the applied voltage. In this case, a lower activation energy related to the junction between interface levels and states in the chains providing the current transport could be observed.

Because of the charge accumulation in the interface states, the potential barrier at the boundary between $\mathrm{SiO}_{2}$ and $\mathrm{Si}$ should inevitably appear. Thus, it is quite natural to suppose that this barrier can control the over-barrier current when the forward voltage becomes higher. At this condition, the current related with over-barrier processes should dominate in the structures. In this 

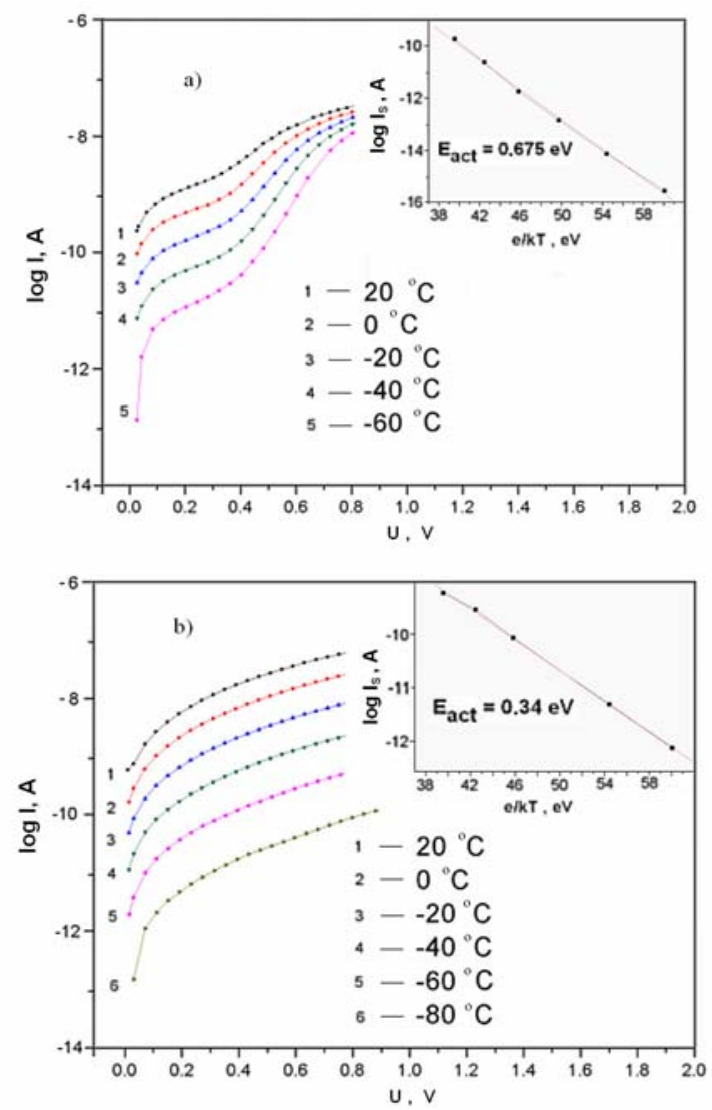

Fig. 5. $I-V$ curves taken for the forward (a) and reverse bias (b).

manner, it could be sequentially explained why the higher activation energy $(\sim 0.6 \mathrm{eV})$ for the sufficiently large forward bias could be obtained in these structures. Both of these current channels are presented in the energy diagram inherent to the investigated structure (Fig. 4).

\subsubsection{Investigation of the thermally stimulated current}

For more detailed investigation of carrier transport mechanisms, the spectra of thermally stimulated current were investigated. This method can be successfully used for many structures with energy traps. The main idea of this method consists in sequential filling and thermally activated discharge processes. The first of these processes is usually caused by an applied voltage at low temperatures for a sufficiently long time. In this work, the initial state of the structure was reached by applying the high reverse bias $-5 \mathrm{~V}$ and cooling the sample down to $-150{ }^{\circ} \mathrm{C}$. It was supposed that all accessible states were filled up by carriers, because the time of exposure with a considerable value of the applied reverse voltage is enough at a sufficiently low initial temperature. A higher time of exposure make no influence on obtaining thermally stimulated currents.

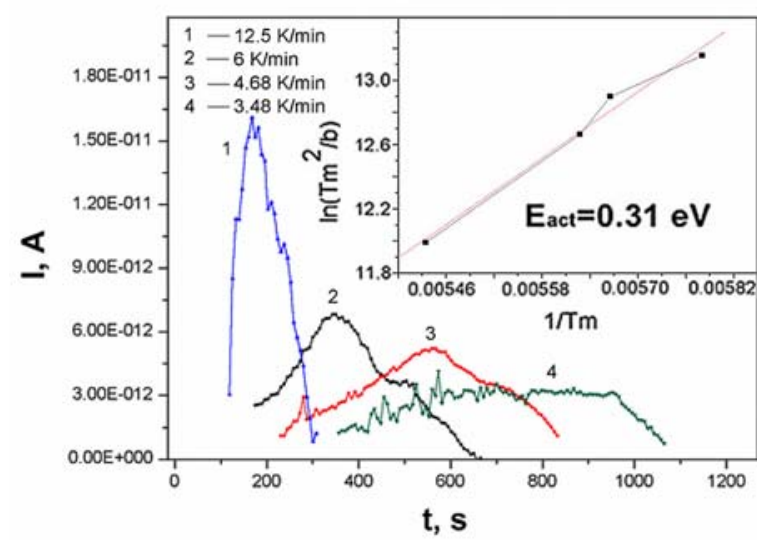

Fig. 6. Thermally stimulated current spectra taken at different speeds of heating with insert for the activation energy determination plot.

The second process was realized practically when the thermally activated discharge current was observed and integrated for several characteristic speeds of heating. Then, in the short current regime the thermally stimulated current was measured. In this paper, we present the results obtained for four various speeds of heating (see Table). The results of this investigation are presented in Fig. 6. The maximums of thermally activated currents were slightly shifted up on the scale of temperatures with decreasing the speed of heating. It reasonably correlates with the main assumption about accumulation of charge in deep interface states in the structure.

In accord with theoretical substantiation of this method given in $[8,9]$, the activation energy of these states $E_{\text {th }}=0.31 \mathrm{eV}$ was calculated using the standard procedure for the number of curves in spectra of Fig 6 . The value of activation energy obtained in this way well coincides with the previous results obtained from the series of $I-V$ curves. This fact shows that the thermally activated current caused the same physical way of carrier escaping from deep interface states through the conductivity chain.

Table. Peak positions taken from the thermally stimulated current spectra for various speeds of heating.

\begin{tabular}{|c|c|}
\hline $\begin{array}{c}\text { Speed of heating } \beta, \\
{ }^{\circ} \mathrm{C} / \mathrm{min}\end{array}$ & $\begin{array}{c}\text { Peak position } T_{m}, \\
{ }^{\circ} \mathrm{C}\end{array}$ \\
\hline 12.5 & -89 \\
\hline 6 & -95.3 \\
\hline 4.68 & -96.5 \\
\hline 3.48 & -100 \\
\hline
\end{tabular}


The area under the thermally activated current curves was calculated, which gave the value of stored charge in insulator. It is noteworthy that the value of this charge is sufficiently large and approximately the same for four various speeds of heating $Q=1.8 \times 10^{-9} \mathrm{Coul}$. It means that all the experiments on thermal stimulation correctly operate with the same volume of the stored charge. At the same time, the noticeable difference between positions of maximums shows that discharge of these states essentially depends on the intensity of escaping process from the interface states. It is indirect evidence that detailed investigation of thermally activated escaping process can give qualitatively new information about specific kinetic inherent to reaching the equilibrium in structures with $\mathrm{Si}$ nanoclusters in $\mathrm{SiO}_{2}$.

\section{Conclusions}

Using the automated experimental setup, we performed investigation of stationary and dynamic measured $C-V$ characteristics and temperature dependences of $I-V$ curves for $\mathrm{Al}_{-} \mathrm{SiO}_{2}-(\mathrm{Si}-\mathrm{ncs})-\mathrm{SiO}_{2}-\mathrm{Si}$ structures with $\mathrm{Si}$ nanoclusters thermally formed by high temperature annealing of $\mathrm{SiO}_{x}$ with $x<2$.

High-frequency capacitance measured in stationary and dynamic modes reveal clearly observed specific differences that can be explained as caused by the influence of charge accumulation on the energy levels of interface states between $\mathrm{Si}$ nanoclusters and $\mathrm{SiO}_{2}$ placed near the quasi-Fermi level position. As it follows from the experimental results, the differences between the stationary and dynamic capacitances appear because of the long-time relaxation process of recharging the states with a sufficiently high density, which are related to the interfaces of nanoclusters.

Being based on the analysis of temperature dependences of $I-V$ curves, the main mechanisms of current transport for the investigated structures were determined. The detailed research of thermally stimulated currents enabled us to calculate the activation energy for the supposedly appearing interface states in $\mathrm{Al}-\mathrm{SiO}_{2}-(\mathrm{Si}-\mathrm{ncs})-\mathrm{SiO}_{2}-\mathrm{Si}$ structures, which are responsible for the accumulation of charge as well as estimate its value $\left(Q=1.8 \times 10^{-9} \mathrm{Coul}\right)$. The data obtained from $I-V$ curves and spectra of the thermally stimulated current conform well with those described in literature. The physical model of accumulation and recharging processes observed in the investigated structures is offered in the paper to explain the experimental results.

\section{Acknowledgements}

This work was supported by Grant №M/90-2010 of Ministry for Education and Science of Ukraine.

\section{References}

1. I. Crupi, D. Corso, G. Ammendola, S. Lombardo, C. Geradi, B. DeSalvo, G. Ghibaudo, E. Rimini, and M. Melanotte, Peculiar aspects of nanocrystal memory cells: Data and extrapolations // IEEE Trans. Nanotechnology 2(4), p. 319-323 (2003).

2. S. Oda, Charge storage in silicon nanocrystals and device application // Mater. Sci. Eng. B 101, p. 1924 (2003).

3. G.A. Kachurin, V.A. Volodin, D.I. Tetelbaum, D.V. Marin, A.F. Leier, A.K. Gutakovsky, A.G. Cherkov, A.N. Mikhailov, Formation of silicon nanocrystals in $\mathrm{SiO}_{2}$ layers by implantation of Si ions with intermediate annealing // Fizika tekhnika poluprovodnikov, 39(5), p. 582-586 (2005), in Russian.

4. V.N. Bogomolov, V.G. Golubev, N.A. Feoktistov, A production of regular three-dimensional lattices of silicon clusters of submicron sizes in $\mathrm{SiO}_{2}$ matrix // Zhurnal tekhnich. fiziki 24(8), p. 90-95 (1998), in Russian.

5. V.A. Dan'ko, I.Z. Indutnyy, V.S. Lysenko, I.Yu. Maidanchuk, V.I. Min'ko, A.N. Nazarov, A.S. Tkachenko, P.E. Shepeliavyi, Kinetics of structural-phase transformations in thin $\mathrm{SiO}_{\mathrm{x}}$ films in the course of rapid thermal annealing // Fizika tekhnika poluprovodnikov 39(10), p. 1239-1245 (2005), in Russian.

6. S.V. Bunak, V.V. Ilchenko, O.V. Tretyak, A.G. Shkavro, Automated installation of investigation of electrophysical parameters of nanoheterostructures // Visnyk Kyivs'kogo Universytetu. Ser. fiz.-mat. nauky, No.3, p. 223-227 (2007), in Ukrainian.

7. S.V. Bunak, A.A. Buyanin, V.V. Ilchenko, V.V. Marin, V.P. Melnik, I.M. Khacevich, O.V. Tretyak, A.G. Shkavro, Electrical properties of semiconductor structures with Si nanoclusters in $\mathrm{SiO}_{2}$ grown by high temperature annealing technology of $\mathrm{SiO}_{\mathrm{x}}$ layer, $\mathrm{x}<2$ // Semiconductor Physics, Quantum Electronics \& Optoelectronics, 13(1), p. 12-18 (2010).

8. V.N. Vetropahov, E.G. Salmon, Thermally Stimulated Current in Non-organic Substance. Nauka, Novosibirsk, 1979, p. 333 (in Russian).

9. S.G. Rzaev, E.P. Nahmedov // Fizika tverdogo tela 35, p. 1083 (1993), in Russian.

10. S.V. Bunak, V.V. Ilchenko, V.P. Melnik, O.V. Tretyak, A.G. Shkavro, I.M. Khacevich, Investigation of electrophysical parameters of a MIS-structure with Si-nanoclusters // Proc. 4-th Intern. Sci. Conf. "Ukrainian Scientific Conference of Semiconductor Physics" “USCSP-4”, Ukraine, Zaporizhzhya, September 15-19, 2009, p. 47-48. 